\title{
A deficiency of apoptosis inducing factor (AIF) in Harlequin mouse heart mitochondria paradoxically reduces $\mathrm{ROS}$ generation during ischemia-reperfusion
}

\author{
Qun Chen ${ }^{1}{ }^{*}$, Karol Szczepanek ${ }^{1}$, Ying Hu ${ }^{1}$, Jeremy Thompson ${ }^{1}$ and Edward J. Lesnefsky ${ }^{1,2,3}$ \\ 1 Division of Cardiology, Department of Internal Medicine, Pauley Heart Center, Virginia Commonwealth University, Richmond, VA, USA \\ ${ }^{2}$ Department of Biochemistry and Molecular Biology, Virginia Commonwealth University, Richmond, VA, USA \\ ${ }^{3}$ McGuire Department of Veterans Affairs Medical Center, Richmond, VA, USA
}

Edited by:

Miguel A. Aon, Johns Hopkins

University School of Medicine, USA

Reviewed by:

Paul S. Brookes, University of

Rochester, USA

Nazareno Paolocci, Johns Hopkins

University, USA

Shey-Shing Sheu, University of

Rochester, USA

\section{*Correspondence:}

Qun Chen, Division of Cardiology, Department of Internal Medicine, Virginia Commonwealth University, 1101 East Marshall Street, PO Box 980281, Richmond, VA 23298, USA e-mail: qchen8@vcu.edu
Background and Aims: AIF (apoptosis inducing factor) is a flavin and NADH containing protein located within mitochondria required for optimal function of the respiratory chain. AlF may function as an antioxidant within mitochondria, yet when released from mitochondria it activates caspase-independent cell death. The Harlequin (Hq) mouse has a markedly reduced content of AIF, providing an experimental model to query if the main role of AIF in the exacerbation of cell death is enhanced mitochondrial generation of reactive oxygen species (ROS) or the activation of cell death programs. We asked if the ROS generation is altered in $\mathrm{Hq}$ heart mitochondria at baseline or following ischemia-reperfusion (IR).

Methods: Buffer perfused mouse hearts underwent $30 \mathrm{~min}$ ischemia and $30 \mathrm{~min}$ reperfusion. Mitochondrial function including oxidative phosphorylation and $\mathrm{H}_{2} \mathrm{O}_{2}$ generation was measured. Immunoblotting was used to determine the contents of AIF and PAR [poly(ADP-ribose)] in cell fractions.

Results: There were no differences in the release of $\mathrm{H}_{2} \mathrm{O}_{2}$ between wild type (WT) and $\mathrm{Hq}$ heart mitochondria at baseline. IR increased $\mathrm{H}_{2} \mathrm{O}_{2}$ generation from WT but not from $\mathrm{Hq}$ mitochondria compared to corresponding time controls. The complex I activity was decreased in WT but not in Hq mice following IR. The relocation of AIF from mitochondria to nucleus was increased in WT but not in Hq mice. IR activated PARP-1 only in WT mice. Cell injury was decreased in the Hq mouse heart following in vitro IR.

Conclusion: A deficiency of AIF within mitochondria does not increase ROS production during IR, indicating that AIF functions less as an antioxidant within mitochondria. The decreased cardiac injury in Hq mouse heart accompanied by less AIF translocation to the nucleus suggests that AIF relocation, rather than the AIF content within mitochondria, contributes to cardiac injury during IR.

Keywords: reactive oxygen species, electron transport chain, apoptosis, poly(ADP-ribose)

\section{INTRODUCTION}

Apoptosis inducing factor (AIF) is a nuclear encoded protein synthesized as a $67 \mathrm{kDa}$ precursor (Sevrioukova, 2011; Natarajan and Becker, 2012). The mature form of AIF ( $62 \mathrm{kDa}$ ) is located within the mitochondrial intermembrane space following import and removal of the mitochondrial localization signal (Sevrioukova, 2011; Natarajan and Becker, 2012). AIF has a pro-survival role when it is located within mitochondria, whereas release of AIF from mitochondria into cytosol followed by nuclear import activates caspase-independent cell death (Sevrioukova, 2011; Natarajan and Becker, 2012). The presence of FAD and NAD cofactors in the mature AIF renders it a potential antioxidant within the mitochondrial intermembrane space (Klein et al., 2002; van Empel et al., 2005), although this concept has been challenged (Sevrioukova, 2011). Nonetheless, the presence of these cofactors strongly suggests that AIF responses both within mitochondria and following release are likely responsive to and modulated by the local redox environment (Sevrioukova, 2011). The lower expression of AIF in Harlequin ( $\mathrm{Hq}$ ) mice impacts metabolism and response to tissue stress, in an organ-dependent manner (Klein et al., 2002; Vahsen et al., 2004). AIF deletion is embryonic lethal (Klein et al., 2002). When activated following birth, AIF deletion in heart and skeletal muscle leads to profound dilated cardiomyopathy and muscle wasting due to a marked decrease in electron transport complex I activity concomitant with increased reactive oxygen species (ROS) production from mitochondria (Joza et al., 2005; Pospisilik et al., 2007). Attenuation of the severity of AIF deficiency through extensive backcross breeding or the use of (female) heterozygotes leads to more modest defects in mitochondrial respiration that do not 
exhibit increased ROS production in the baseline state, and in fact display potential resistance to exogenous disease (Pospisilik et al., 2007) suggestive of protective modulation of metabolism (Chen et al., 2007; Oxler et al., 2012). Thus, consistent with observations in other genetic models of partial complex I deficiency, a protective response to tissue injury may be observed (Oxler et al., 2012).

The $\mathrm{Hq}$ mouse exhibits an approximately $80 \%$ decrease in AIF content in all tissues. Defects are most profound in brain, with decreased complex I activity and complex I dependent respiration (Klein et al., 2002) and the development of disease in retina and brain (Hisatomi et al., 2001), reminiscent of human mitochondrial disease (Sevrioukova, 2011). In contrast, in heart, the metabolic defects are more subtle, although increased cardiac injury following in vivo ischemia-reperfusion (IR) and an increased susceptibility to heart failure in an aortic banding model of cardiac pressure overload have been reported (van Empel et al., 2005). The capacity to scavenge ROS is reported to be decreased in Hq mouse heart mitochondria compared to wild type (van Empel et al., 2005), suggesting that AIF has a potential antioxidant role. However, the net release of $\mathrm{H}_{2} \mathrm{O}_{2}$ is not altered in $\mathrm{Hq}$ mouse brain mitochondria compared to wild type (Chinta et al., 2009). This finding does not support an antioxidant role for AIF within mitochondria.

Cardiac mitochondria provide the energy to support heart function, whereas diseased and disabled mitochondria are a source of cardiomyocyte damage (Lesnefsky et al., 2001; Gustafsson and Gottlieb, 2008; Murphy and Steenbergen, 2008). IR results in damage to the electron transport chain that serves as a key source of ROS that exacerbate cardiac injury (Turrens, 2003; Chen et al., 2007). The net release of ROS from intact mitochondria represents a balance between ROS generation and the capacity of mitochondrial antioxidants (Turrens et al., 1991; Rigobello et al., 2006; Wenzel et al., 2008; Stanley et al., 2011). In the present study, we investigated if the net release of ROS from Hq mouse heart mitochondria is increased compared to wild type at baseline and if the genetic knockdown of AIF in Hq mice affects the net ROS generation and susceptibility to injury in the heart following the tissue stress of IR.

Translocation of AIF from mitochondria to the nucleus triggers caspase-independent cell death by inducing DNA damage (Yu et al., 2002; Sevrioukova, 2011; Natarajan and Becker, 2012). The mature form of AIF is anchored in the inner mitochondrial membrane (Ozaki et al., 2007; Chen et al., 2011). IR leads to a release of AIF from mitochondria into cytosol in isolated mouse heart, whereas administration of a calpain inhibitor prevents the loss of AIF from mitochondria (Chen et al., 2011). These results support that activation of mitochondrial localized $\mu$-calpain is required to detach the AIF from the inner membrane (Ozaki et al., 2007; Chen et al., 2011) and indicate that retention of AIF within mitochondria provides cardioprotection during IR (Chen et al., 2011). The protection through retention of AIF within mitochondria can be due to a potential antioxidant role of the AIF or the prevention of AIF translocation to the nucleus. In the present study, mitochondria and nucleus were isolated from buffer perfused hearts to evaluate if IR increases the AIF translocation from mitochondria to nucleus. In the in vivo IR model, the length of the ischemic period was limited in that a relative long ischemic period could trigger a fatal arrhythmia occurrence in $\mathrm{Hq}$ mice (van Empel et al., 2005). Thus, a buffer perfused heart model was selected in the present study to avoid this confounding issue and to allow an ischemic period resulting in a moderate extent of mitochondrial and cardiac damage, relevant to in situ IR. Hq mice provide an experimental model to test the potential contribution of AIF to local mitochondrial protection compared to the deleterious cellular effects of nuclear translocation during IR.

\section{METHODS}

The experimental procedures conformed to the Guide for the Care and Use of Laboratory Animals and were approved by the Institutional Animal Care and Use Committees of Virginia Commonwealth University (VCU) and the McGuire Department of Veterans Affairs Medical Center.

\section{PREPARATION OF MOUSE HEART FOR PERFUSION}

Wild type or $\mathrm{Hq}$ mice [2-3 months of age (22-28 g), male] were anesthetized with pentobarbital sodium (100 mg/g i.p.) and anti-coagulated with heparin (1 IU/g i.p.) (Chen et al., 2011). In this study, only male mice were used because Hq mice had a gender-dependent response in an experimental stroke model (Yuan et al., 2009). Compared to male $\mathrm{Hq}$ mice, female $\mathrm{Hq}$ mice exhibited markedly decreased brain injury after experimental stroke (Yuan et al., 2009). Since the AIF gene is located on the $\mathrm{X}$ chromosome, using male mice will avoid gender-dependent gene dosage effects on cardioprotection. Hearts were excised and retrograde perfused via the aorta in the Langendorff mode (constant pressure, $75 \mathrm{mmHg}$ ) with modified Krebs-Henseleit buffer (composition, in mM: $115 \mathrm{NaCl}, 4.0 \mathrm{KCl}, 2.0 \mathrm{CaCl}_{2}, 25 \mathrm{NaHCO}_{3}$, $1.1 \mathrm{MgSO}_{4} \cdot \mathrm{H}_{2} \mathrm{O}, 0.9 \mathrm{KH}_{2} \mathrm{PO}_{4}$, and 5.5 glucose) oxygenated with $95 \% \mathrm{O}_{2}+5 \% \mathrm{CO}_{2}$. Cardiac function was monitored with a balloon inserted into the left ventricle using Powerlab (AD Instruments, Colorado Springs, $\mathrm{CO}$ ). Heart rate was maintained at $420 \mathrm{bpm}$ with pacing during the equilibration period. Pacing was stopped during global ischemia and restored at $15 \mathrm{~min}$ reperfusion. In the IR group, hearts were buffer-perfused for $15 \mathrm{~min}$, followed by $30 \mathrm{~min}$ global ischemia at $37^{\circ} \mathrm{C}$ within $30 \mathrm{~min}$ reperfusion (mitochondrial isolation) or $1 \mathrm{~h}$ reperfusion (infarction measurement). In the time control group, hearts were bufferperfused without IR. Myocardial infarct size was determined using TTC staining (Chen et al., 2006). Coronary effluent was collected to determine LDH activity in each group (Chen et al., 2006).

\section{ISOLATION OF CARDIAC MITOCHONDRIA FROM THE MOUSE HEART WITH PROTEASE}

Trypsin was used in the mitochondrial isolation protocol in order to remove potential contamination from AIF located in the cytosol (Chen et al., 2011). The mouse heart was harvested and immediately placed in cold buffer A [100 mM KCl, $50 \mathrm{mM}$ 3-(N-morpholino) propanesulfonic acid (MOPS), 1 mM EGTA, $5 \mathrm{mM} \mathrm{MgSO}_{4}, 1 \mathrm{mM}$ ATP]. Cardiac tissue was homogenized with a polytron at $10,000 \mathrm{rpm}$ and incubated with trypsin $(5 \mathrm{mg} / \mathrm{g})$ for 15 min. Cold buffer B ( $0.2 \%$ BSA + buffer A) was then added into the homogenate. The homogenate was centrifuged at $500 \times \mathrm{g}$ for 
$10 \mathrm{~min}$. The supernatant was centrifuged at $3000 \times \mathrm{g}$ for $10 \mathrm{~min}$. to pellet mitochondria. The mitochondrial pellet was washed and suspended in $100 \mathrm{mM} \mathrm{KCl,} 50 \mathrm{mM}$ MOPS, and 0.5 mM EGTA.

\section{ISOLATION OF NUCLEUS FROM BUFFER-PERFUSED MOUSE HEARTS}

The polytron pellet was used to isolate nuclear components using a commercial kit from Thermo Scientific (Pittsburgh, PA, catalog \# 78835) using supplied solutions and differential centrifugation according to instructions provided.

\section{MEASUREMENT OF OXIDATIVE PHOSPHORYLATION AND ENZYME ACTIVITIES IN ISOLATED MITOCHONDRIA}

Oxygen consumption by mitochondria was measured using a Clark-type oxygen electrode at $30^{\circ} \mathrm{C}$ using glutamate + malate (complex I substrate) or succinate + rotenone (complex II substrate) as donors (Lesnefsky et al., 1997). Respiratory enzyme activities [NADH-decylubiquinol oxidoreductase, rotenone sensitive (complex I)]; NADH ferricyanide oxioreductase (NFR, flavoprotein portion of complex I); Succinate-decylubiquinone oxidoreductase (complex II); and citrate synthase were measured in detergent solubilized mitochondria according to the method of Dr. Hoppel as previously described (Krahenbuhl et al., 1991; Lesnefsky et al., 1997; Chen et al., 2008).

\section{DETECTION OF $\mathrm{H}_{2} \mathrm{O}_{2}$ PRODUCTION FROM ISOLATED MOUSE HEART MITOCHONDRIA}

$\mathrm{H}_{2} \mathrm{O}_{2}$ production by isolated mitochondria was measured using the oxidation of the fluorogenic indicator Amplex red in the presence of horseradish peroxidase without exogenous SOD (Chen et al., 2003). Glutamate + malate and succinate + rotenone were used as complex I and complex II substrates, respectively. Rotenone and antimycin A were used to detect the maximal $\mathrm{H}_{2} \mathrm{O}_{2}$ generation from complex I and complex III, respectively.

\section{DETERMINATION OF MITOCHONDRIAL CALCIUM RETENTION CAPACITY}

Mitochondrial calcium retention capacity (CRC) was used to reflect opening of the mitochondrial permeability transition pore (MPTP) in isolated mitochondria (Chen et al., 2012a). CRC was studied in the single cell fluorimeter (PerkinElmer, Waltham, Massachusetts) using repetitive calcium pulses (Chen et al., 2012a). Freshly isolated mitochondria $(0.25 \mathrm{mg})$ were incubated in buffer ( $150 \mathrm{mM}$ sucrose, $50 \mathrm{mM} \mathrm{KCl,} 2 \mathrm{mM} \mathrm{KPi}$, and $20 \mathrm{mM}$ Tris/ $\mathrm{HCl}, \mathrm{pH} 7.4$ ) for $90 \mathrm{~s}$ with stirring at $30^{\circ} \mathrm{C}$ with $0.5 \mu \mathrm{M}$ calcium green. Succinate $(5 \mathrm{mM})$ was used as substrate. Pulses of calcium ( 5 nmoles) were added at $60 \mathrm{~s}$ intervals. The number of pulses that resulted in calcium release indicated the onset of MPTP.

\section{WESTERN BLOT ANALYSIS}

Particle free cytosol, purified mitochondria, and nucleus were boiled for $5 \mathrm{~min}$. in buffer including 4\% (w/v) SDS, $1 \mathrm{mM} 2-$ mercaptoethanol, $10 \mathrm{mM}$ Tris/ $\mathrm{HCl}(\mathrm{pH}$ 6.8) and $10 \%(\mathrm{w} / \mathrm{v})$ glycerol. Equal amounts of protein were loaded onto 4-15\% or $4-20 \%$ SDS-PAGE (dependent on molecular weight of proteins), electrophoresed and transferred to a PVDF membrane.
The membranes were first blocked by $5 \%$ non-fat milk for $1 \mathrm{~h}$ followed by exposure to primary antibodies overnight (Chen et al., 2011). Antibodies to AIF, PARP-1, GAPDH, lamin, and subunit IV of cytochrome oxidase were purchased from Cell Signaling Technology (Danvers, MA). Monoclonal PAR antibody was purchased from Millipore (Billerica, MA). The blots were incubated with peroxidase conjugated anti-rabbit or anti-mouse secondary antibody for $1 \mathrm{~h}$ prior to ECL detection (GE Healthcare Life Science, Pittsburgh, PA). The intensity of blotting was quantified by Fuji Film Image station (Edison, NJ).

\section{STATISTICAL ANALYSIS}

Data were expressed as the mean \pm standard error of the mean. Differences among four groups were compared by one-way analysis of variance with post-hoc comparisons performed using the Student-Newman-Keuls test of multiple comparisons (Sigmastat 3.5, ProgramPaketet, Gothenburg, Sweden). Differences in CRC and $\mathrm{H}_{2} \mathrm{O}_{2}$ between wild type and $\mathrm{Hq}$ mice were compared by unpaired student $t$-test. A difference of $p<0.05$ was considered significant.

\section{RESULTS \\ IR DECREASED OXIDATIVE PHOSPHORYLATION IN BOTH WILD TYPE AND Hq HEART MITOCHONDRIA}

There were no differences in the rate of oxidative phosphorylation between time control wild type and $\mathrm{Hq}$ mitochondria when glutamate + malate (complex I) or succinate + rotenone (complex II) were used as substrates. IR decreased the ADP-stimulated respiration in mitochondria from both wild type and Hq using either substrate (Table 1). The rate of dinitrophenol (DNP) uncoupled respiration was also decreased in both wild type and $\mathrm{Hq}$ mice following IR (Table 1), supporting that IR damages the electron transport chain. Interestingly, the rate of oxidative phosphorylation with glutamate + malate in $\mathrm{Hq}$ hearts was decreased following IR compared to corresponding wild type (Table 1), whereas the rate of succinate oxidation was similar in $\mathrm{Hq}$ and wild type hearts following IR (Table 1). These results indicated that

Table 1 | The rate of oxidative phosphorylation in wild type and $\mathrm{Hq}$ mitochondria following IR.

\begin{tabular}{|c|c|c|c|c|c|c|}
\hline Mice & $n$ & State 3 & State 4 & RCR & ADP/O & DNP \\
\hline \multicolumn{7}{|c|}{$\begin{array}{l}\text { GLUTAMATE + MALATE WAS USED AS COMPLEX I SUBSTRATE } \\
\text { (nAO/min/mg PROTEIN) }\end{array}$} \\
\hline WT-TC & $N=8$ & $324 \pm 11$ & $56 \pm 5$ & $6.2 \pm 0.8$ & $2.73 \pm 0.108$ & $341 \pm 18$ \\
\hline WT-IR & $N=8$ & $271 \pm 10^{*}$ & $56 \pm 4$ & $5.1 \pm 0.5$ & $2.80 \pm 0.09$ & $281 \pm 11^{*}$ \\
\hline $\mathrm{Hq}-\mathrm{TC}$ & $N=6$ & $315 \pm 27$ & $51 \pm 9$ & $6.7 \pm 0.8$ & $2.80 \pm 0.08$ & $321 \pm 40$ \\
\hline $\mathrm{Hq}-\mathrm{IR}$ & $N=7$ & $224 \pm 12^{*} \dagger$ & $52 \pm 9$ & $5.3 \pm 1.1$ & $2.76 \pm 0.13$ & $229 \pm 16^{*} \dagger$ \\
\hline \multicolumn{7}{|c|}{$\begin{array}{l}\text { SUCCINATE + ROTENONE WAS USED AS COMPLEX II SUBSTRATE } \\
\text { (nAO/min/mg PROTEIN) }\end{array}$} \\
\hline WT-TC & $N=8$ & $474 \pm 14$ & $144 \pm 3$ & $3.3 \pm 0.1$ & $2.00 \pm 0.07$ & $435 \pm 12$ \\
\hline WT-IR & $N=8$ & $266 \pm 11^{*}$ & $133 \pm 6$ & $2.0 \pm 0.1^{*}$ & $1.84 \pm 0.05$ & $339 \pm 14^{*}$ \\
\hline $\mathrm{Hq}-\mathrm{TC}$ & $N=6$ & $496 \pm 20$ & $133 \pm 18$ & $3.4 \pm 0.2$ & $2.08 \pm 0.06$ & $471 \pm 30$ \\
\hline $\mathrm{Hq}-\mathrm{IR}$ & $N=7$ & $268 \pm 15^{*}$ & $124 \pm 6$ & $2.2 \pm 0.1^{*}$ & $2.07 \pm 0.09$ & $346 \pm 25^{*}$ \\
\hline
\end{tabular}

Mean \pm s.e.m. ${ }^{*} p<0.05$ vs. corresponding time control $(T C) ;{ }^{t} p<0.05$ vs. WT-IR (ischemia-reperfusion). RCR, respiratory control ratio. DNP $(0.3 \mathrm{mM})$, dinitrophenol to measure the rate of uncoupled respiration. 
IR led to additional decreases in respiration in $\mathrm{Hq}$ mouse heart mitochondria when NADH-dependent substrates were used.

\section{IR DECREASED COMPLEX I ACTIVITY IN WILD TYPE BUT NOT Hq MOUSE HEART MITOCHONDRIA}

In order to test if IR led to further damage to complex I; NADH:decylubiquinol oxidoredutase, NFR, and complex II activities were measured. Complex I activity [shown as the ratio of complex I/CS (citrate synthase), Table 2] was decreased in wild type following IR compared to time control. However, complex I activity was not decreased in $\mathrm{Hq}$ mitochondria following IR compared to its corresponding time control. The NADH dehydrogenase activity (NFR) was not decreased in either wild type or $\mathrm{Hq}$ mice following IR (Table 2), consistent with previous study (Chen et al., 2007; Szczepanek et al., 2011). NADH dehydrogenase activity was slightly higher in $\mathrm{Hq}$ mouse heart mitochondria following IR compared to corresponding wild type (Table 2). The physiological significance of this subtle difference is unclear. IR did not alter complex II activity in wild type or Hq mice (Table 2). These results support that IR leads to a complex I defect in wild type mouse heart mitochondria.

\section{THE GENERATION OF $\mathrm{H}_{2} \mathrm{O}_{2}$ WAS DECREASED IN Hq MOUSE HEART MITOCHONDRIA FOLLOWING IR}

There were no differences in $\mathrm{H}_{2} \mathrm{O}_{2}$ generation between time control wild type and $\mathrm{Hq}$ heart mitochondria using complex I (Figure 1A) or complex II substrates (Figure 1C). IR markedly increased the production of $\mathrm{H}_{2} \mathrm{O}_{2}$ in wild type but not in $\mathrm{Hq}$ mouse heart mitochondria with either a complex I or complex II substrate (Figures 1A,C). Compared to time control, inhibition of complex I using rotenone dramatically increased $\mathrm{H}_{2} \mathrm{O}_{2}$ generation in wild type mouse heart following IR (Figure 1B). In contrast, rotenone inhibition did not increase the $\mathrm{H}_{2} \mathrm{O}_{2}$ generation in $\mathrm{Hq}$ mouse heart following IR (Figure 1B). The maximal ROS generation from mitochondria was induced with antimycin $\mathrm{A}$ inhibition. Inhibition of complex III using antimycin A increased the $\mathrm{H}_{2} \mathrm{O}_{2}$ generation in both wild type and $\mathrm{Hq}$ mouse heart following IR vs. time control (Figure 1D). However, there were no differences in ROS generation between wild type and $\mathrm{Hq}$ mice with or without IR.

\section{CARDIAC INJURY WAS DECREASED IN Hq MOUSE HEART FOLLOWING IR}

In the buffer perfused hearts, myocardial injury was decreased in the Hq mouse heart following IR compared to wild type. Knock down of AIF content in $\mathrm{Hq}$ mouse heart did not affect the cardiac function before ischemia (Figure 2A). IR decreased left ventricular developed pressure (LVDP) in both wild type and Hq hearts vs. time control. Systolic function was improved in $\mathrm{Hq}$ hearts vs. wild type during reperfusion (Figure 3A). Strikingly, the infarct size was also much smaller in $\mathrm{Hq}$ mice than in wild type (Figure $2 \mathbf{B}$ ). The release of $\mathrm{LDH}$ into coronary effluent was much lower in $\mathrm{Hq}$ mice than in wild type (Figure 2C), also indicating less necrosis.

\section{IR DECREASED THE CRC IN BOTH WILD TYPE AND Hq MOUSE HEART MITOCHONDRIA}

The CRC (Figure 3A) was decreased in mitochondria from nonischemic Hq mice compared to wild type (Figure 3B), suggesting that a decrease in AIF content within mitochondria sensitizes to calcium-stimulated MPTP opening. Although there was a slight difference in the CRC between $\mathrm{Hq}$ and wild type following IR (Figures 3A,B), the small magnitude of this difference may not exert a significant impact on cardiac injury during IR.

\section{IR ACTIVATED PARP-1 IN WILD TYPE BUT NOT IN Hq MICE}

Activation of PARP-1 during IR increases the generation of PAR [Poly (ADP-ribose) (PAR)] that is transferred to cytosol and mitochondria (Sevrioukova, 2011). The content of PAR was markedly increased in wild type following IR compared to time control (Figures 4A-C), indicating that IR leads to PARP-1 activation. In contrast, IR did not alter the PAR content in Hq mice following IR (Figures 4A-C).

\section{IR DECREASED THE AIF CONTENT WITHIN MITOCHONDRIA AND INCREASED NUCLEAR AIF CONTENT IN WILD TYPE MICE}

The precursor of AIF $(67 \mathrm{kd})$ is nuclear-encoded and subsequently transported into the mitochondrial matrix via its mitochondrial targeting sequence (Sevrioukova, 2011). The mature form of AIF $(62 \mathrm{kd})$ is formed in the matrix through cleavage of precursor protein via a mitochondrial matrix peptidase (Sevrioukova, 2011). The mature AIF (62 kd) is transferred into the mitochondrial intermembrane space through the Tim23 protein (Sevrioukova, 2011). Consistent with these concepts, two AIF bands are detected in non-ischemic wild type mouse heart mitochondria (Figure 4D). In contrast, the mature form of AIF $(62 \mathrm{kd})$ is almost undetectable in $\mathrm{Hq}$ mouse heart mitochondria (Figure 4D), confirming the lower content of AIF within mitochondria. The content of AIF within mitochondria was decreased in wild type mice following IR compared to time control (Figure 4D). The AIF content $(62 \mathrm{kd})$ in nucleus was increased in wild type hearts following IR (Figure 4E).

Table 2 | The enzyme activities of Complex I and II in wild type and Hq mitochondria with and without IR.

\begin{tabular}{|c|c|c|c|c|c|c|}
\hline Mice & $n$ & CS & Complex I/CS & NFR/CS & ClI/CS & $\mathrm{Cll}+\mathrm{Q} / \mathrm{CS}$ \\
\hline WT-IR & $N=8$ & $2937 \pm 1.9$ & $0.142 \pm 0.010^{*}$ & $0.542 \pm 0.029$ & $0.059 \pm 0.008$ & $0.142 \pm 0.012$ \\
\hline $\mathrm{Hq}-\mathrm{IR}$ & $N=7$ & $2545 \pm 1.7$ & $0.174 \pm 0.019$ & $0.793 \pm 0.081^{\dagger}$ & $0.039 \pm 0.010$ & $0.103 \pm 0.027$ \\
\hline
\end{tabular}

Mean \pm s.e.m. ${ }^{*} p<0.05$ vs. WT-TC; ${ }^{\dagger} p<0.05$ vs. WT-IR. NFR, NADH dehydrogenase; CS, citrate synthase. Complex II activity (CII) was determined in the presence and absence of exogenous decylubiquinone (Q). 

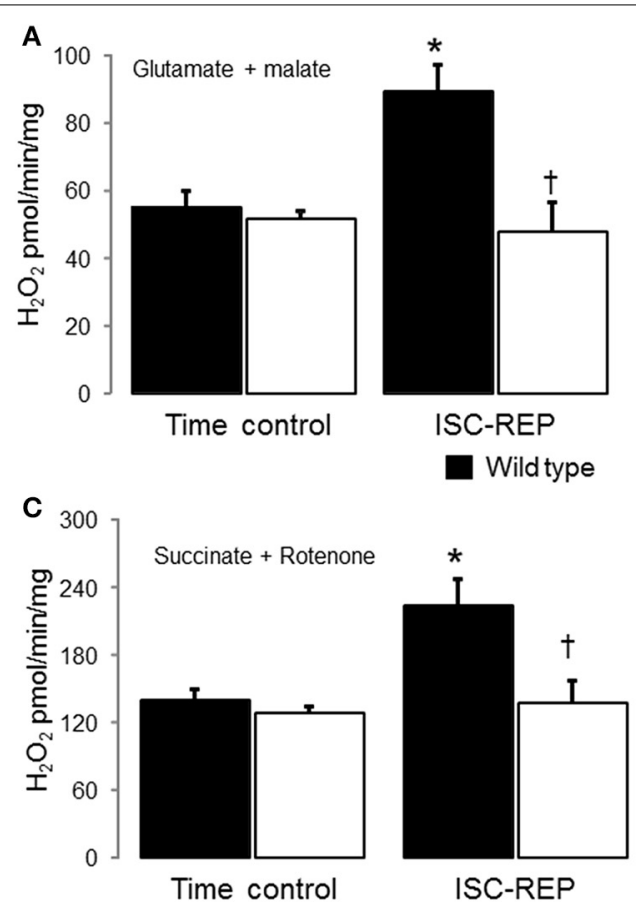

FIGURE 1 | Ischemia-reperfusion (IR) increases the net $\mathrm{H}_{2} \mathrm{O}_{2}$ production in wild type but not in $\mathrm{Hq}$ mouse cardiac mitochondria. There were no differences in the net release of $\mathrm{H}_{2} \mathrm{O}_{2}$ between wild type and Harlequin mouse heart mitochondria from non-ischemic hearts. IR increased the generation of $\mathrm{H}_{2} \mathrm{O}_{2}$ in wild type but not in Harlequin mice compared to time control (A). The maximal $\mathrm{H}_{2} \mathrm{O}_{2}$ generation from complex I was measured using rotenone to inhibit complex I. An AIF deficiency in Harlequin mice did not alter the maximal $\mathrm{H}_{2} \mathrm{O}_{2}$ generation from complex I compared to wild type in control heart (B). The maximal

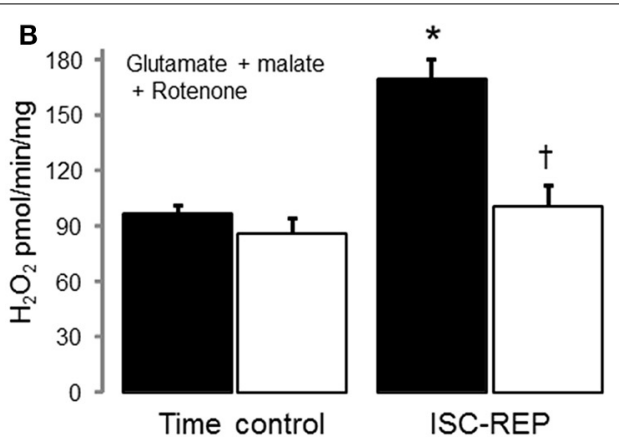

Harlequin

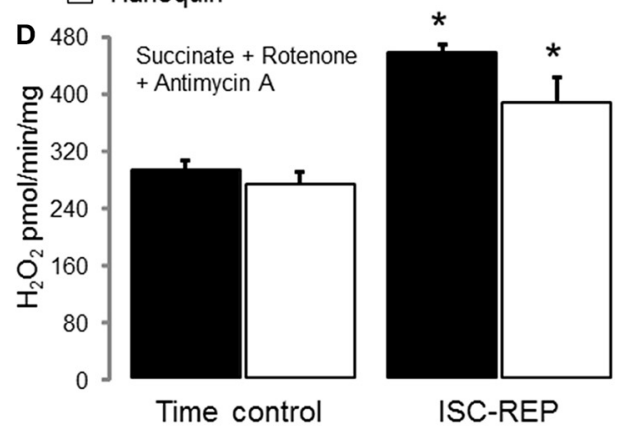

$\mathrm{H}_{2} \mathrm{O}_{2}$ generation from complex I was also decreased in Harlequin mice following IR compared to wild type (B). Knock down of AIF in Harlequin mice did not alter the $\mathrm{H}_{2} \mathrm{O}_{2}$ generation using succinate + rotenone as complex II substrates compared to wild type control. The $\mathrm{H}_{2} \mathrm{O}_{2}$ generation was decreased in Harlequin mice following IR compared to wild type (C). The maximal $\mathrm{H}_{2} \mathrm{O}_{2}$ generation from complex III in the presence of antimycin A was not decreased in Harlequin mice following IR compared to wild type (D). Data are expressed as mean \pm s.e.m.; ${ }^{*} p<0.05$ vs. time control; ${ }^{\dagger} p<0.05$ vs. wild type IR.

\section{DISCUSSION}

In the present study, a deficiency of AIF within $\mathrm{Hq}$ mouse heart mitochondria does not increase the net release of $\mathrm{H}_{2} \mathrm{O}_{2}$ compared to wild type, consistent with reports in brain mitochondria. IR leads to increased net release of ROS from wild type heart mitochondria compared to non-ischemic controls. In contrast, the net release of ROS from $\mathrm{Hq}$ heart mitochondria is unchanged following IR. Thus, genetic knockdown of AIF within mitochondria does not increase the net release of ROS from the electron transport chain. These findings suggest that AIF less likely functions as a key mitochondrial antioxidant in the heart, especially following the stress of IR. The PARP-1 is activated in wild type but not in $\mathrm{Hq}$ mice following IR. Translocation of the AIF from mitochondria to the nucleus is increased in wild type but not in $\mathrm{Hq}$ mice following IR. The decrease in cardiac injury in $\mathrm{Hq}$ mouse heart accompanied by less AIF translocation to the nucleus suggests that the amount of AIF that relocates to the nucleus, rather than the AIF content within mitochondria, is the key factor that contributes to cardiac injury during IR.

\section{COMPLEX I DAMAGE DURING IR}

Complex I activity is decreased in heart mitochondria following both in vivo (Rouslin and Millard, 1980; Rouslin, 1983) and in vitro IR (Lesnefsky et al., 2001; Gustafsson and Gottlieb, 2008;
Murphy and Steenbergen, 2008). In the present study, IR leads to decreased complex I activity without alteration in NADH dehydrogenase activity (NFR). These results indicate that ischemia likely damages complex I at the iron sulfur centers (Chen et al., 2008) distal to the flavoprotein, in line with previous studies (Ohnishi and Trumpower, 1980; Chen et al., 2006; Zhou et al., 2006; Szczepanek et al., 2011).

Oxidative modification of complex I by nitrosation (Burwell et al., 2006) or glutathionylation (Hurd et al., 2008) or the modification of its inner membrane environment via depletion of cardiolipin (Paradies et al., 2004) all contribute to decreases in activity. Mitochondrial AIF content also affects complex I activity, especially in brain and retina (Klein et al., 2002; van Empel et al., 2005). Depletion of AIF also decreases complex I activity in heart mitochondria (Pospisilik et al., 2007). However, the effect of lower expression of AIF in Hq mice on heart mitochondrial complex I activity is not consistent (Szczepanek et al., 2013). Thus, an AIF deficiency may affect complex I activity in a tissue-dependent manner. Genetic depletion of PARP-1 protects complex I activity in mouse heart following IR (Zhou et al., 2006), indicating that PARP-1 activation contributes to the complex I defect during IR. In the present study, IR decreases complex I activity accompanied by an activated PARP-1 in wild type mice. In contrast, IR does not decrease complex I activity in Hq mice. PARP-1 is 


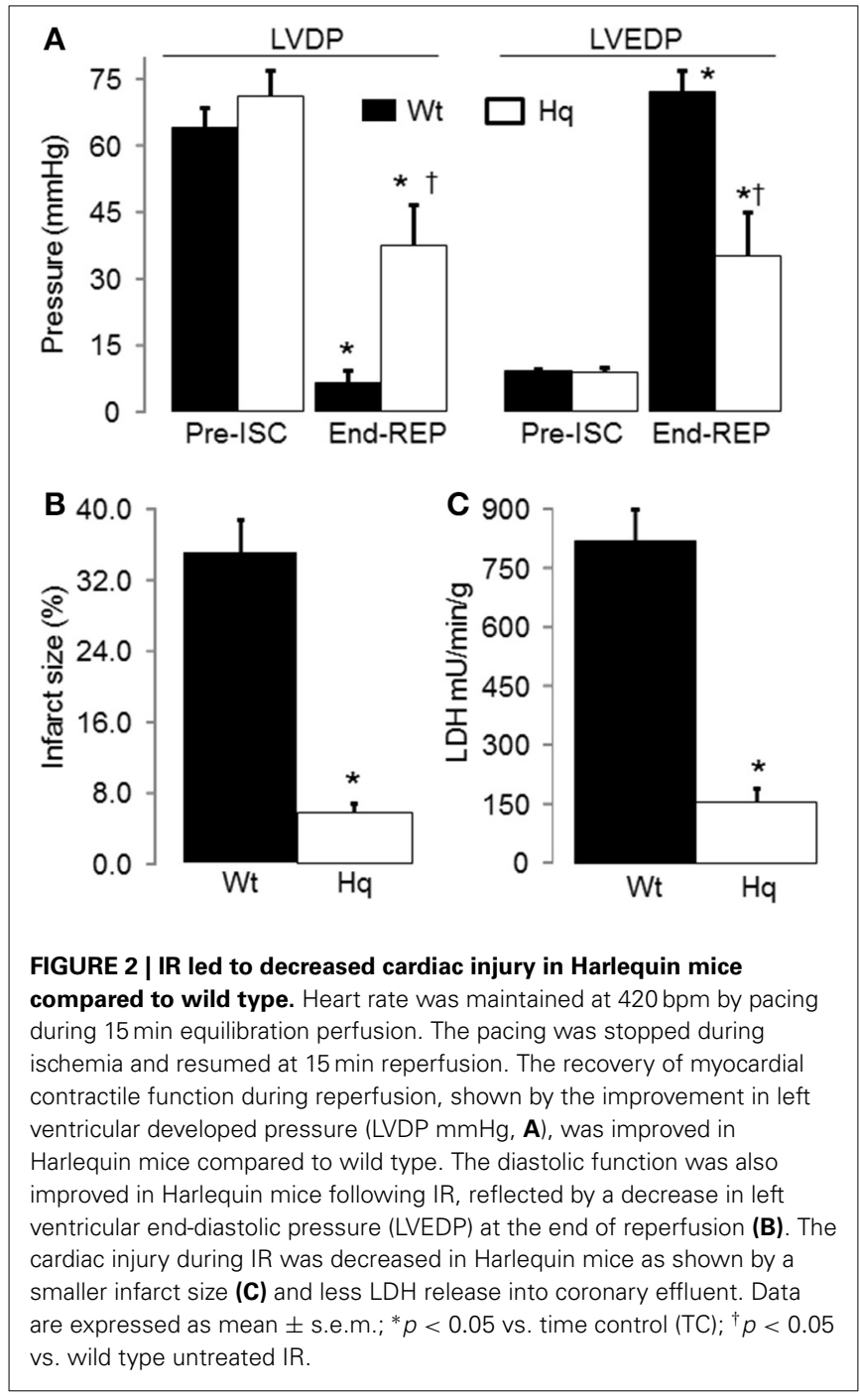

also not activated in $\mathrm{Hq}$ mice following IR. These results support that activation of PARP-1 contributes to the complex I defect during IR. Since PARP-1 is considered as a nuclear protein, complex I inhibition by PARP-1 activation appears to present a challenge (Figure 5) (Zhou et al., 2006). Recently, a mitochondrial localized PARP-1 has been identified (Rossi et al., 2009). Thus, activation of PARP-1 may directly regulate complex I activity (Figure 5).

\section{COMPLEX I INHIBITION AND CARDIOPROTECTION DURING IR}

The ischemia-damaged respiratory chain including complex I, is a key source of ROS that increases cardiac injury (Turrens, 2003; Chen et al., 2007). Blockade of proximal electron transport using amobarbital before ischemia protects complex I and decreases cardiac injury during reperfusion, supporting that preservation of complex I activity reduces cardiac injury (Chen et al., 2006). Transient partial (Xu et al., 2014) or complete (Stewart et al., 2009; Chen et al., 2012b) blockade of complex I at the onset of reperfusion decreases myocardial injury in buffer perfused hearts, indicating that a temporary complex I inhibition is also beneficial for cardiac recovery. However, persistent, severe complex I
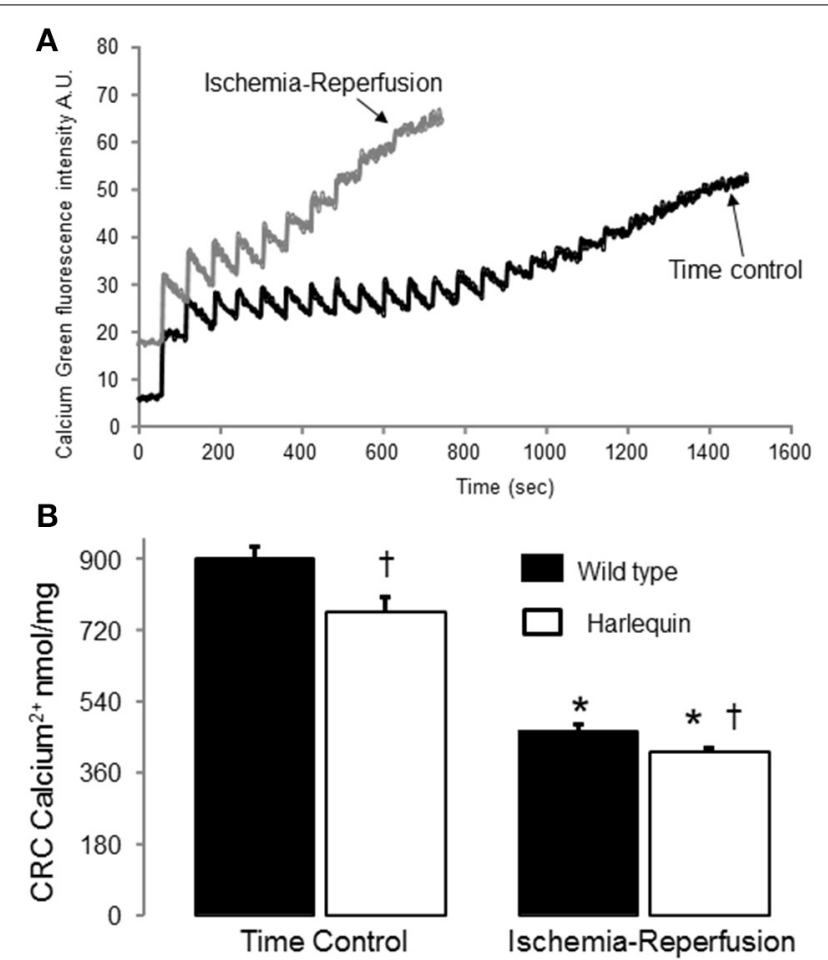

FIGURE 3 | IR decreased the CRC in wild type and Harlequin cardiac mitochondria. Original tracings of CRC measurement in wild type heart mitochondria were shown in panel (A). The CRC was decreased in control Harlequin mouse heart mitochondria compared to wild type (B), suggesting that the sensitivity to MPTP opening was increased in Harlequin mice in the basal condition. IR led to decreased CRC in both wild type and Harlequin mice compared to time control (B). Compared to wild type, IR led to a slight further decrease in CRC in Harlequin mice (B). Data are expressed as mean \pm s.e.m.; ${ }^{*} p<0.05$ vs. time control; ${ }^{\dagger} p<0.05$ vs. wild type IR.

inhibition is detrimental to the heart both at baseline and for recovery during reperfusion (Karamanlidis et al., 2013). In contrast to wild type, the complex I activity is not altered in $\mathrm{Hq}$ mice following IR. The lack of damage to complex I during IR in Hq mice may lead to decreased ROS generation that contributes to the observed decrease in cardiac injury in $\mathrm{Hq}$ mice. Alteration of mitochondrial antioxidants including thioredoxin reductase2 significantly affects a release of $\mathrm{H}_{2} \mathrm{O}_{2}$ from mitochondria. The IR-induced complex I damage may also increase $\mathrm{H}_{2} \mathrm{O}_{2}$ by inhibiting thioredoxin reductase- 2 through alteration of its redox state (Rigobello et al., 2006; Horstkotte et al., 2011; Stanley et al., 2011). The increased oxidative stress will favor activated $\mu$-calpain to cleave AIF and facilitate its release from mitochondria (Norberg et al., 2010).

\section{TRANSLOCATION OF AIF FROM MITOCHONDRIA TO NUCLEUS INCREASES CARDIAC INJURY DURING IR}

The mature form of AIF $(62 \mathrm{kd})$ is anchored at the inner mitochondrial membrane within the intermembrane space (Otera et al., 2005). Release of AIF from the mitochondria and translocation to the nucleus to activate caspase-independent cell death is a multistep process. First, the mature AIF bound within mitochondria on the inner membrane requires liberation. Cleavage of 


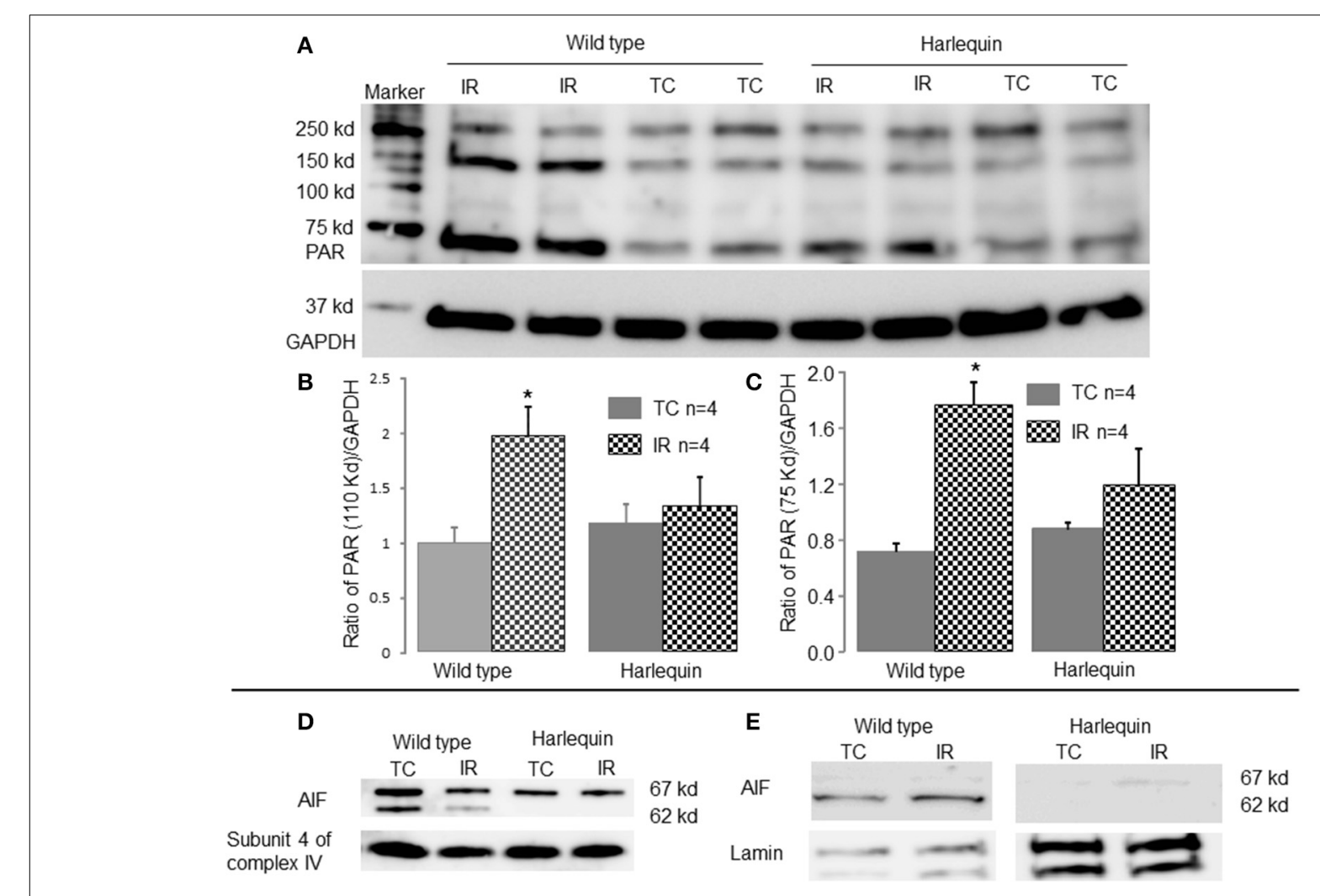

FIGURE 4 | IR increases AIF translocation from mitochondria to nucleus in wild type. The content of poly (ADP-ribose) (PAR) was markedly increased in wild type mice following IR compared to time control, indicating that IR activated PARP-1 (A-C). There were no differences in the PAR content in $\mathrm{Hq}$ mice between time control and mice following IR (A-C). GAPDH was used as a cytosol marker for loading control. The precursor form of AIF (67 kd) and the mature form of AIF ( $62 \mathrm{kd})$ were detected in wild type mouse heart mitochondria (D). Compared to control, IR markedly decreased the content of the mature form of AIF (62 kd band) in wild type mice (D). The mature AIF content in Harlequin mice was of course decreased compared to wild type (D). In wild type, IR increased AIF content (62 kd) in nucleus compared to time control, indicating a translocation of AIF from mitochondria to nucleus (E). Subunit 4 of cytochrome oxidase and lamin were used as protein loading control for mitochondria and nucleus, respectively. the mature $62 \mathrm{kd}$ form of AIF by activated mitochondrial calpains (Ozaki et al., 2007), $t$-bid (Cabon et al., 2012) or other proteases can liberate AIF from the inner membrane, with release of a truncated, approximately $57 \mathrm{kd}$ AIF peptide. Next, permeation of the outer mitochondrial membrane is required for AIF release (Ozaki et al., 2007). IR increases MPTP opening as a mechanism of increased outer membrane permeability (Weiss et al., 2003), with oxidative stress is a key contributor to the increased susceptibility to MPTP opening during IR (Weiss et al., 2003; Halestrap et al., 2004; Chen et al., 2012a). In contrast, activation of PARP-1 is not involved in the permeation of the outer mitochondrial membrane during IR (Schriewer et al., 2013). The decreased AIF content in the purified mitochondria following IR supports that IR does lead to a loss of AIF from mitochondria. The increased MPTP opening in wild type mice during reperfusion favors a release of AIF from mitochondria into cytosol with subsequent translocation to the nucleus. It currently appears that even following MPTP, the released AIF is the $57 \mathrm{kd}$ cleaved form, at least based upon calcium activation of mitochondrial calpains concomitant with MPTP. This area of calcium mediated injury deserves further consideration. As discussed above, IR activates PARP-1 in wild type but not Hq mice (Pacher and Szabo, 2007). Although activation of PARP-1 provides a beneficial effect to repair DNA damage, over activation of PARP-1 has a detrimental effect via consumption of $\mathrm{NAD}^{+}$(Pacher and Szabo, 2007). PAR, which is generated by activation of PARP-1 within nucleus, is released into cytosol and subsequently relocates to mitochondria to induce AIF release from mitochondria (Pacher and Szabo, 2007). Interestingly, a portion of the mature AIF is also reported to be loosely attached on the mitochondrial outer membrane (Yu et al., 2006, 2009). The PAR can detach the AIF from the outer membrane (Figure 5) (Wang et al., 2009). Outer membrane bound-AIF has been identified in mouse heart mitochondria (Chen and Lesnefsky, unpublished data). Thus, activation of PARP-1 may increase AIF translocation to the nucleus through detachment of mature AIF from the outer membrane, in addition to release of the pool from the inner membrane via cleavage. The accumulation of AIF in the nucleus accompanied by increased cardiac injury in wild type mice following IR supports the proposal that the translocation of AIF from the mitochondria to the nucleus augments cardiac injury.

\section{GENETIC INHIBITION OF AIF EXPRESSION IN Hq MICE DECREASES CARDIAC INJURY DURING IR}

Although cardiac injury is increased in Hq mouse heart following in vivo IR compared to wild type controls (van Empel et al., 2005), cardiac injury is actually decreased in Hq mouse heart 


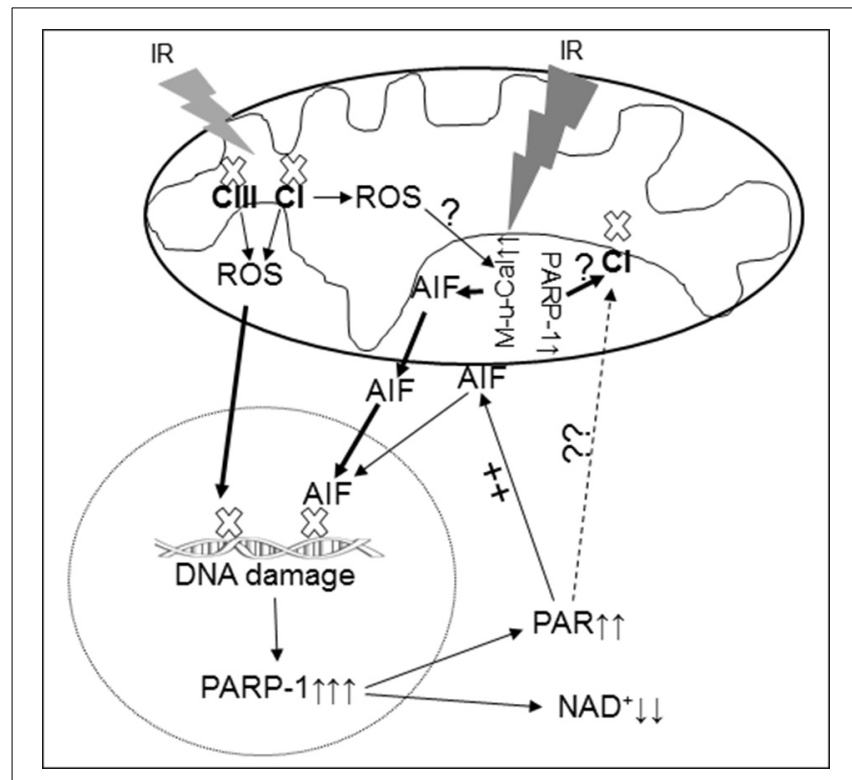

FIGURE 5 | Depiction of the PARP-1 activation and AIF translocation during IR. IR damages the electron transport chain and increases the ROS generation from complex I and complex III and activates mitochondrial $\mu$-calpain (M- $\mu$-Cal) to induce release of AIF from the inner mitochondrial membrane. Translocation of AIF from mitochondria to nucleus enhances DNA damage and PARP-1 activation, in turn further reducing NAD ${ }^{+}$content. Oxidative stress during IR leads to DNA damage DNA that activates the PARP-1 that leads to decreased NAD ${ }^{+}$content in cytosol and increased generation of PAR within nucleus. The PAR is released into cytosol and relocates to mitochondria and facilitates AIF release from the outer mitochondrial membrane. The activated PARP-1 may contribute to complex I defect through an indirect mechanism. IR may activate mitochondrial localized PARP-1 to induce direct complex I damage in cardiac mitochondria. $\mathrm{Cl}$, complex I; CIII, complex III; M- $\mu$-Cal, mitochondrial $\mu$-calpain.

following in vitro IR. Several key differences likely contribute to these divergent results. In the present study, only male $\mathrm{Hq}$ mice were used based upon the rationale discussed in Methods, whereas both female and male $\mathrm{Hq}$ mice were included in the previous in vivo study (van Empel et al., 2005). This is important, since gender-related cardiac protection as well as gene dosage issues related to the $\mathrm{X}$ chromosome location of the aif gene can introduce variability. In the present study, only 2-3-month-old mice were used whereas middle-aged and elderly mice were used in the in vivo study (van Empel et al., 2005). In vivo, there is the additional impact of exogenous inflammatory cells (with or without AIF deficiency). Furthermore, in vivo, substrate utilization is uncontrolled. The metabolism of fatty acids in vivo, in contrast to glucose utilization in the current study, may have exacerbated the phenotype of mitochondrial defects present. Taken together, gender, age, exogenous cells and the different IR models likely resulted in the differences observed in our current study compared to the previous in vivo study. An isolated heart was used in order to focus on myocyte specific responses in the present study.

In summary, the key contribution of AIF to cardiac injury during IR is related to release from mitochondria and activation of programmed cell death via cytosolic transport, nuclear import and DNA cleavage. The findings in cardiac mitochondria from $\mathrm{Hq}$ mice compared to littermate controls support that a decreased content of AIF does not enhance ROS production from mitochondria nor augment cardiac injury at baseline nor during IR. Thus, AIF does not exert significant mitochondrial antioxidant protection during IR. The prevention of AIF translocation to nucleus is a potentially powerful approach to reduce cardiac injury.

\section{ACKNOWLEDGMENTS}

This work was supported by a Scientist Development Grant (11SDG5120011) from the American Heart Association (Qun Chen) and the Office of Research and Development, Medical Research Service, Department of Veterans Affairs (Edward J. Lesnefsky), and the Pauley Heart Center, Virginia Commonwealth University.

\section{REFERENCES}

Burwell, L. S., Nadtochiy, S. M., Tompkins, A. J., Young, S., and Brookes, P. S. (2006). Direct evidence for S-nitrosation of mitochondrial complex I. Biochem. J. 394(Pt 3), 627-634. doi: 10.1042/BJ20051435

Cabon, L., Galan-Malo, P., Bouharrour, A., Delavallee, L., Brunelle-Navas, M. N., Lorenzo, H. K., et al. (2012). BID regulates AIF-mediated caspase-independent necroptosis by promoting BAX activation. Cell Death Diff. 19, 245-256. doi: 10.1038/cdd.2011.91

Chen, Q., Camara, A. K., Stowe, D. F., Hoppel, C. L., and Lesnefsky, E. J. (2007). Modulation of electron transport protects cardiac mitochondria and decreases myocardial injury during ischemia and reperfusion. Am. J. Physiol. Cell Physiol. 292, C137-C147. doi: 10.1152/ajpcell.00270.2006

Chen, Q., Moghaddas, S., Hoppel, C. L., and Lesnefsky, E. J. (2006). Reversible blockade of electron transport during ischemia protects mitochondria and decreases myocardial injury following reperfusion. J. Pharmacol. Exp. Ther. 319, 1405-1412. doi: 10.1124/jpet.106.110262

Chen, Q., Moghaddas, S., Hoppel, C. L., and Lesnefsky, E. J. (2008). Ischemic defects in the electron transport chain increase the production of reactive oxygen species from isolated rat heart mitochondria. Am. J. Physiol. Cell Physiol. 294, C460-C466. doi: 10.1152/ajpcell.00211.2007

Chen, Q., Paillard, M., Gomez, L., Li, H., Hu, Y., and Lesnefsky, E. J. (2012a). Postconditioning modulates ischemia-damaged mitochondria during reperfusion. J. Cardiovasc. Pharmacol. 59, 101-108. doi: 10.1097/FJC.0b013e31823827cc

Chen, Q., Paillard, M., Gomez, L., Ross, T., Hu, Y., Xu, A., et al. (2011). Activation of mitochondrial mu-calpain increases AIF cleavage in cardiac mitochondria during ischemia-reperfusion. Biochem. Biophys. Res. Commun. 415, 533-538. doi: 10.1016/j.bbrc.2011.10.037

Chen, Q., Ross, T., Hu, Y., and Lesnefsky, E. J. (2012b). Blockade of electron transport at the onset of reperfusion decreases cardiac injury in aged hearts by protecting the inner mitochondrial membrane. J. Aging Res. 2012:753949. doi: 10.1155/2012/753949

Chen, Q., Vazquez, E. J., Moghaddas, S., Hoppel, C. L., and Lesnefsky, E. J. (2003). Production of reactive oxygen species by mitochondria: central role of complex III. J. Biol. Chem. 278, 36027-36031. doi: 10.1074/jbc.M304854200

Chinta, S. J., Rane, A., Yadava, N., Andersen, J. K., Nicholls, D. G., and Polster, B. M. (2009). Reactive oxygen species regulation by AIF- and complex I-depleted brain mitochondria. Free Radic. Biol. Med. 46, 939-947. doi: 10.1016/j.freeradbiomed.2009.01.010

Gustafsson, A. B., and Gottlieb, R. A. (2008). Heart mitochondria: gates of life and death. Cardiovasc. Res. 77, 334-343. doi: 10.1093/cvr/cvm005

Halestrap, A. P., Clarke, S. J., and Javadov, S. A. (2004). Mitochondrial permeability transition pore opening during myocardial reperfusion-a target for cardioprotection. Cardiovasc. Res. 61, 372-385. doi: 10.1016/S0008-6363(03)00533-9

Hisatomi, T., Sakamoto, T., Murata, T., Yamanaka, I., Oshima, Y., Hata, Y., et al. (2001). Relocalization of apoptosis-inducing factor in photoreceptor apoptosis induced by retinal detachment in vivo. Am. J. Pathol. 158, 1271-1278. doi: 10.1016/S0002-9440(10)64078-3

Horstkotte, J., Perisic, T., Schneider, M., Lange, P., Schroeder, M., Kiermayer, C., et al. (2011). Mitochondrial thioredoxin reductase is essential for 
early postischemic myocardial protection. Circulation 124, 2892-2902. doi: 10.1161/CIRCULATIONAHA.111.059253

Hurd, T. R., Requejo, R., Filipovska, A., Brown, S., Prime, T. A., Robinson, A. J., et al. (2008). Complex I within oxidatively stressed bovine heart mitochondria is glutathionylated on Cys-531 and Cys-704 of the $75-\mathrm{kDa}$ subunit: potential role of CYS residues in decreasing oxidative damage. J. Biol. Chem. 283, 24801-24815. doi: 10.1074/jbc.M803432200

Joza, N., Oudit, G. Y., Brown, D., Benit, P., Kassiri, Z., Vahsen, N., et al. (2005). Muscle-specific loss of apoptosis-inducing factor leads to mitochondrial dysfunction, skeletal muscle atrophy, and dilated cardiomyopathy. Mol. Cell. Biol. 25, 10261-10272. doi: 10.1128/MCB.25.23.10261-10272.2005

Karamanlidis, G., Lee, C. F., Garcia-Menendez, L., Kolwicz, S. C. Jr., Suthammarak, W., Gong, G., et al. (2013). Mitochondrial complex I deficiency increases protein acetylation and accelerates heart failure. Cell Metab. 18, 239-250. doi: 10.1016/j.cmet.2013.07.002

Klein, J. A., Longo-Guess, C. M., Rossmann, M. P., Seburn, K. L., Hurd, R. E., Frankel, W. N., et al. (2002). The harlequin mouse mutation downregulates apoptosis-inducing factor. Nature 419, 367-374. doi: 10.1038/nature01034

Krahenbuhl, S., Chang, M., Brass, E. P., and Hoppel, C. L. (1991). Decreased activities of ubiquinol:ferricytochrome c oxidoreductase (complex III) and ferrocytochrome c:oxygen oxidoreductase (complex IV) in liver mitochondria from rats with hydroxycobalamin[c-lactam]-induced methylmalonic aciduria. J. Biol. Chem. 266, 20998-21003.

Lesnefsky, E. J., Moghaddas, S., Tandler, B., Kerner, J., and Hoppel, C. L. (2001). Mitochondrial dysfunction in cardiac disease: ischemia-reperfusion, aging, and heart failure. J. Mol. Cell. Cardiol. 33, 1065-1089. doi: 10.1006/jmcc.2001.1378

Lesnefsky, E. J., Tandler, B., Ye, J., Slabe, T. J., Turkaly, J., and Hoppel, C. L. (1997). Myocardial ischemia decreases oxidative phosphorylation through cytochrome oxidase in subsarcolemmal mitochondria. Am. J. Physiol. 273(3 Pt 2), H1544H1554.

Murphy, E., and Steenbergen, C. (2008). Mechanisms underlying acute protection from cardiac ischemia-reperfusion injury. Physiol. Rev. 88, 581-609. doi: 10.1152/physrev.00024.2007

Natarajan, S. K., and Becker, D. F. (2012). Role of apoptosis-inducing factor, proline dehydrogenase, and NADPH oxidase in apoptosis and oxidative stress. Cell Health Cytoskelet. 2012, 11-27. doi: 10.2147/CHC.S4955

Norberg, E., Gogvadze, V., Vakifahmetoglu, H., Orrenius, S., and Zhivotovsky, B. (2010). Oxidative modification sensitizes mitochondrial apoptosis-inducing factor to calpain-mediated processing. Free Radic. Biol. Med. 48, 791-797. doi: 10.1016/j.freeradbiomed.2009.12.020

Ohnishi, T., and Trumpower, B. L. (1980). Differential effects of antimycin on ubisemiquinone bound in different environments in isolated succinate. cytochrome c reductase complex. J. Biol. Chem. 255, 3278-3284.

Otera, H., Ohsakaya, S., Nagaura, Z., Ishihara, N., and Mihara, K. (2005). Export of mitochondrial AIF in response to proapoptotic stimuli depends on processing at the intermembrane space. EMBO J. 24, 1375-1386. doi: 10.1038/sj.emboj.7600614

Oxler, E. M., Dolga, A., and Culmsee, C. (2012). AIF depletion provides neuroprotection through a preconditioning effect. Apoptosis 17, 1027-1038. doi: 10.1007/s10495-012-0748-8

Ozaki, T., Tomita, H., Tamai, M., and Ishiguro, S. (2007). Characteristics of mitochondrial calpains. J. Biochem. 142, 365-376. doi: 10.1093/jb/mvm143

Pacher, P., and Szabo, C. (2007). Role of poly(ADP-ribose) polymerase 1 (PARP-1) in cardiovascular diseases: the therapeutic potential of PARP inhibitors. Cardiovasc. Drug Rev. 25, 235-260. doi: 10.1111/j.1527-3466.2007.00018.x

Paradies, G., Petrosillo, G., Pistolese, M., Di Venosa, N., Federici, A., and Ruggiero, F. M. (2004). Decrease in mitochondrial complex I activity in ischemic/reperfused rat heart: involvement of reactive oxygen species and cardiolipin. Circ. Res. 94, 53-59. doi: 10.1161/01.RES.0000109416.56608.64

Pospisilik, J. A., Knauf, C., Joza, N., Benit, P., Orthofer, M., Cani, P. D., et al. (2007). Targeted deletion of AIF decreases mitochondrial oxidative phosphorylation and protects from obesity and diabetes. Cell 131, 476-491. doi: 10.1016/j.cell.2007.08.047

Rigobello, M. P., Vianello, F., Folda, A., Roman, C., Scutari, G., and Bindoli, A. (2006). Differential effect of calcium ions on the cytosolic and mitochondrial thioredoxin reductase. Biochem. Biophys. Res. Commun. 343, 873-878. doi: 10.1016/j.bbrc.2006.03.050

Rossi, M. N., Carbone, M., Mostocotto, C., Mancone, C., Tripodi, M., Maione, R., et al. (2009). Mitochondrial localization of PARP-1 requires interaction with mitofilin and is involved in the maintenance of mitochondrial DNA integrity. J. Biol. Chem. 284, 31616-31624. doi: 10.1074/jbc.M109.025882

Rouslin, W. (1983). Mitochondrial complexes I, II, III, IV, and V in myocardial ischemia and autolysis. Am. J. Physiol. 244, H743-H748.

Rouslin, W., and Millard, R. W. (1980). Canine myocardial ischemia: defect in mitochondrial electron transfer complex I. J. Mol. Cell. Cardiol. 12, 639-645. doi: 10.1016/0022-2828(80)90021-8

Schriewer, J. M., Peek, C. B., Bass, J., and Schumacker, P. T. (2013). ROS-mediated PARP activity undermines mitochondrial function after permeability transition pore opening during myocardial ischemia-reperfusion. J. Am. Heart Assoc. 2:e000159. doi: 10.1161/JAHA.113.000159

Sevrioukova, I. F. (2011). Apoptosis-inducing factor: structure, function, and redox regulation. Antioxid. Redox Signal. 14, 2545-2579. doi: 10.1089/ars.2010.3445

Stanley, B. A., Sivakumaran, V., Shi, S., McDonald, I., Lloyd, D., Watson, W. H., et al. (2011). Thioredoxin reductase-2 is essential for keeping low levels of $\mathrm{H}(2) \mathrm{O}(2)$ emission from isolated heart mitochondria. J. Biol. Chem. 286, 33669-33677. doi: 10.1074/jbc.M111.284612

Stewart, S., Lesnefsky, E. J., and Chen, Q. (2009). Reversible blockade of electron transport with amobarbital at the onset of reperfusion attenuates cardiac injury. Transl. Res. 153, 224-231. doi: 10.1016/j.trsl.2009.02.003

Szczepanek, K., Chen, Q., Derecka, M., Salloum, F. N., Zhang, Q., Szelag, M., et al. (2011). Mitochondrial-targeted Signal transducer and activator of transcription 3 (STAT3) protects against ischemia-induced changes in the electron transport chain and the generation of reactive oxygen species. J. Biol. Chem. 286, 29610-29620. doi: 10.1074/jbc.M111.226209

Szczepanek, K., Hu, Y., Lesnefsky, E. J., and Chen, Q. (2013). Deficiency of Apoptosis inducing factor (AIF) decreases complex I activity and increases the ROS generation in isolated cardiac mitochondria. Faseb. J. 27, 1085.18(abstract).

Turrens, J. F. (2003). Mitochondrial formation of reactive oxygen species. J. Physiol. 552(Pt 2), 335-344. doi: 10.1113/jphysiol.2003.049478

Turrens, J. F., Beconi, M., Barilla, J., Chavez, U. B., and McCord, J. M. (1991). Mitochondrial generation of oxygen radicals during reoxygenation of ischemic tissues. Free Radic. Res. Commun. 12-13(Pt 2), 681-689. doi: $10.3109 / 10715769109145847$

Vahsen, N., Cande, C., Briere, J. J., Benit, P., Joza, N., Larochette, N., et al. (2004). AIF deficiency compromises oxidative phosphorylation. EMBO J. 23, 4679-4689. doi: 10.1038/sj.emboj.7600461

van Empel, V. P., Bertrand, A. T., van der Nagel, R., Kostin, S., Doevendans, P. A., Crijns, H. J., et al. (2005). Downregulation of apoptosis-inducing factor in harlequin mutant mice sensitizes the myocardium to oxidative stress-related cell death and pressure overload-induced decompensation. Circ. Res. 96, e92-e101. doi: 10.1161/01.RES.0000172081.30327.28

Wang, Y., Kim, N. S., Li, X., Greer, P. A., Koehler, R. C., Dawson, V. L., et al. (2009). Calpain activation is not required for AIF translocation in PARP-1-dependent cell death (parthanatos). J. Neurochem. 110, 687-696. doi: 10.1111/j.14714159.2009.06167.x

Weiss, J. N., Korge, P., Honda, H. M., and Ping, P. (2003). Role of the mitochondrial permeability transition in myocardial disease. Circ. Res. 93, 292-301. doi: 10.1161/01.RES.0000087542.26971.D4

Wenzel, P., Schuhmacher, S., Kienhofer, J., Muller, J., Hortmann, M., Oelze, M., et al. (2008). Manganese superoxide dismutase and aldehyde dehydrogenase deficiency increase mitochondrial oxidative stress and aggravate age-dependent vascular dysfunction. Cardiovasc. Res. 80, 280-289. doi: 10.1093/cvr/cvn182

Xu, A., Szczepanek, K., Maceyka, M. W., Ross, T., Bowler, E., Hu, Y., et al. (2014). Transient complex I inhibition at the onset of reperfusion by extracellular acidification decreases cardiac injury. Am. J. Physiol. Cell Physiol. 306, C1142-C1153. doi: 10.1152/ajpcell.00241.2013

Yu, S. W., Andrabi, S. A., Wang, H., Kim, N. S., Poirier, G. G., Dawson, T. M., et al. (2006). Apoptosis-inducing factor mediates poly(ADP-ribose) (PAR) polymer-induced cell death. Proc. Natl. Acad. Sci. U.S.A. 103, 18314-18319. doi: 10.1073/pnas.0606528103

Yu, S. W., Wang, H., Poitras, M. F., Coombs, C., Bowers, W. J., Federoff, H. J., et al. (2002). Mediation of poly(ADP-ribose) polymerase-1-dependent cell death by apoptosis-inducing factor. Science 297, 259-263. doi: 10.1126/science.1072221

Yu, S. W., Wang, Y., Frydenlund, D. S., Ottersen, O. P., Dawson, V. L., and Dawson, T. M. (2009). Outer mitochondrial membrane localization of apoptosisinducing factor: mechanistic implications for release. ASN Neuro 1, 275-281. doi: 10.1042/AN20090046 
Yuan, M., Siegel, C., Zeng, Z., Li, J., Liu, F., and McCullough, L. D. (2009). Sex differences in the response to activation of the poly (ADP-ribose) polymerase pathway after experimental stroke. Exp. Neurol. 217, 210-218. doi: 10.1016/j.expneurol.2009.02.012

Zhou, H. Z., Swanson, R. A., Simonis, U., Ma, X., Cecchini, G., and Gray, M. O. (2006). Poly(ADP-ribose) polymerase-1 hyperactivation and impairment of mitochondrial respiratory chain complex I function in reperfused mouse hearts. Am. J. Physiol. Heart Circ. Physiol. 291, H714-H723. doi: 10.1152/ajpheart.00823.2005

Conflict of Interest Statement: The authors declare that the research was conducted in the absence of any commercial or financial relationships that could be construed as a potential conflict of interest.
Received: 01 May 2014; accepted: 30 June 2014; published online: 22 July 2014.

Citation: Chen Q, Szczepanek K, Hu Y, Thompson J and Lesnefsky EJ (2014) A deficiency of apoptosis inducing factor (AIF) in Harlequin mouse heart mitochondria paradoxically reduces ROS generation during ischemia-reperfusion. Front. Physiol. 5:271. doi: 10.3389/fphys.2014.00271

This article was submitted to Mitochondrial Research, a section of the journal Frontiers in Physiology.

Copyright $\odot 2014$ Chen, Szczepanek, Hu, Thompson and Lesnefsky. This is an openaccess article distributed under the terms of the Creative Commons Attribution License (CC BY). The use, distribution or reproduction in other forums is permitted, provided the original author(s) or licensor are credited and that the original publication in this journal is cited, in accordance with accepted academic practice. No use, distribution or reproduction is permitted which does not comply with these terms. 